\title{
Time damping of linear non-adiabatic magnetoacoustic waves in a slab-like quiescent prominence
}

\author{
J. Terradas ${ }^{1}$, M. Carbonell ${ }^{2}$, R. Oliver ${ }^{1}$, and J. L. Ballester ${ }^{1}$ \\ ${ }^{1}$ Departament de Física, Universitat de les Illes Balears, 07122 Palma de Mallorca, Spain \\ 2 Departament de Matemàtiques i Informàtica, Universitat de les Illes Balears, 07122 Palma de Mallorca, Spain \\ e-mail: [jaume.terradas;marc.carbonell;ramon.oliver;dfsjlb0]@uib.es
}

Received 9 September 2004 / Accepted 5 January 2005

\begin{abstract}
We study the time damping of linear non-adiabatic magnetoacoustic waves in a homogeneous, isothermal and bounded magnetic slab of plasma with physical properties akin to those of quiescent solar prominences. Because of the chosen configuration, our results are related to short or intermediate period prominence oscillations and show that the damping times of fast modes are very long compared to those of slow modes. In an attempt to mimic optically thick prominences, different prominence regimes have been considered by reducing radiative losses. Then, when the temperature and/or density of the prominence are modified, the damping time varies in a complex way which also depends on the prominence regime considered. In all the prominence regimes, a minimum of the damping time can be obtained for a certain value of temperature and density. Finally, the consideration of different heating mechanisms, the case of no heating included, can modify the damping times in a substantial way while the periods are only slightly affected.
\end{abstract}

Key words. Sun: oscillations - Sun: magnetic fields - Sun: corona - Sun: prominences

\section{Introduction}

The study of prominence oscillations goes back to the $1950 \mathrm{~s}$ and 60 s when the first reports on the presence of oscillatory motions in solar filaments were published (e.g. Ramsey \& Smith 1966). From the observational point of view, prominence oscillations can be grossly classified, according to the amplitude of periodic variations, in large and small amplitude oscillations. Small amplitude oscillations, which are the subject of this paper, have velocity amplitudes much smaller than those of large amplitude oscillations, and the detected peak velocity ranges from the noise level (down to $0.1 \mathrm{~km} \mathrm{~s}^{-1}$ in some cases) to $2-3 \mathrm{~km} \mathrm{~s}^{-1}$, although larger values also have been reported (Bashkirtsev \& Mashnich 1984; Molowny-Horas et al. 1999; Terradas et al. 2002). These small amplitude prominence oscillations have been detected mainly in the velocity field and early observational studies revealed a wide range of characteristic periods. The apparent tendency of periods to group below $10 \mathrm{~min}$ or in the range 40-80 min led to the distinction between short- and long-period oscillations. Later, more reports of periods in the range 10-40 min were published (e.g. Yi et al. 1991; Sutterlin et al. 1997; Regnier et al. 2001) and the intermediate-period class emerged. Furthermore, the study of the spatial distribution of the periods of oscillation in a quiescent prominence carried out by Terradas et al. (2002) showed that there are large regions within the prominence body with similar values of the period, i.e. the period is coherent over medium sized areas of the prominence, and the few determinations of the wavelength available up to now give values between 20000 and $65000 \mathrm{~km}$ (Thompson \& Schmieder 1991; Yi \& Engvold 1991; Molowny-Horas et al. 1997; Terradas et al. 2002). Observational evidence of small amplitude prominence oscillations has been summarized in reviews by Oliver (1999), Engvold (2001), Oliver \& Ballester (2002), Wiehr (2004) and Engvold (2004).

Sometimes, the oscillatory amplitude tends to decrease in time in such a way that the periodicity totally disappears after a few periods (e.g. Landman et al. 1997; Tsubaki \& Takeuchi 1986; Wiehr et al. 1989; Molowny-Horas et al. 1999; Terradas et al. 2002). This is then interpreted as a signature of wave damping by some as yet unknown mechanism. The twodimensional analysis of the oscillations of a quiescent prominence made by Terradas et al. (2002) has allowed to study the spatial distribution of the damping of the oscillations and has pointed out that it is a common feature in large areas, especially close to the edge of the prominence structure. Reliable values of the damping time, $\tau_{\mathrm{D}}$, have been derived from the oscillations of two quiescent prominences (Molowny-Horas et al. 1999; Terradas et al. 2002), after fitting a sinusoidal function multiplied by a factor $\exp \left(-t / \tau_{\mathrm{D}}\right)$ to Doppler velocity time series recorded simultaneously in different positions; the damping times have been found to be between two and three times the period. 
From the theoretical point of view, prominence oscillations have been interpreted in terms of linear and adiabatic magnetohydrodynamic (MHD) waves. In particular, many theoretical works have studied the MHD modes of oscillations of slab prominence equilibria and are well summarized in reviews by Oliver (1999, 2001, 2004), Oliver \& Ballester (2002) and Ballester (2003).

The essential difference between the various slab models considered to describe prominence equilibrium (Joarder \& Roberts 1992; Oliver et al. 1993) is the treatment of gravity. When gravity is not included this allows for straight magnetic field lines, while when it is included magnetic field lines are curved. However, despite the different shape of field lines, the main features of the oscillatory spectrum are similar and so the influence of gravity on the properties of the MHD modes is not relevant in this kind of configurations.

Linear non-adiabatic waves can provide interesting physical effects such as time and spatial damping of disturbances (Field 1965), and the most simple approach is to take into consideration a radiative loss term in the energy equation based on Newton's law of cooling with a constant radiative relaxation time $\left(\tau_{\mathrm{R}}\right)$. Then, the modes may have complex frequencies and so perturbations contain a term of the form $\exp \left(-t / \tau_{\mathrm{D}}\right)$, where $\tau_{\mathrm{D}}$ is the damping time. The limit $\tau_{\mathrm{R}} \rightarrow \infty$ corresponds to a perturbation that takes infinite time to damp, i.e. the adiabatic limit, while $\tau_{\mathrm{R}}=0$ corresponds to perturbations which are immediately damped, i.e. the isothermal limit. In the case of a magnetised medium, this approach was used by Webb \& Roberts (1980), who analysed magnetohydrodynamic waves in an unbounded atmosphere in the presence of a uniform vertical magnetic field, while in the case of slab prominence models, the above approach was used by Terradas et al. (2001) to study the radiative damping of oscillations in the Kippenhahn-Schlüter (Kippenhahn \& Schlüter 1957) and Menzel's (Menzel \& Evans 1953) prominence models. Concerning the Kippenhahn-Schlüter $(K-S)$ model, Terradas et al. (2001) found that the fundamental slow mode is almost undamped while its harmonics are strongly damped, while for Menzel's model all the slow modes are strongly damped; in both equilibrium models fast modes are practically undamped. Also, Ballai (2003) has discussed qualitatively the spatial damping of linear magnetoacoustic waves in prominences when different effects such as viscosity, electrical resistivity, thermal conduction, thermal radiation, etc. are taken into account. The conclusions suggest that only waves with very short wavelengths can be damped by thermal conduction, while radiative damping would a viable mechanism only when a very short radiative relaxation time, based on Spiegel's formula, is considered.

A more complete approach to linear non-adiabatic waves can be obtained with the inclusion of different mechanisms representing radiative losses and heating, as well as including thermal conduction. In this regard, Ibañez \& Escalona (1993) studied the spatial damping and amplification of magnetosonic linear waves in an unbounded optically thin plasma with solar abundances in the temperature range $10^{4}-10^{8} \mathrm{~K}$, while Carbonell et al. (2004) have studied the damping time of this kind of waves in an unbounded medium having prominence, prominence-corona transition region and coronal properties. Furthermore, using non-adiabatic MHD waves, several studies have focused on the problem of coronal heating by proposing that such heating can be provided by means of different mechanisms based on wave dissipation (Porter et al. 1994a,b; Laing \& Edwin 1994).

In this work, our aim is to study the damping times of linear non-adiabatic magnetoacoustic waves in a quiescent solar prominence modelled as a homogeneous slab threaded by a constant transverse magnetic field. Then, we consider an energy equation including optically thin radiative losses, thermal conduction, as well as different expressions, representing various mechanisms, for the heating term. Taking into account the assumed configuration, these results could be applied to short and intermediate-period magnetoacoustic modes of a slab-like quiescent prominence.

The layout of the paper is as follows: in Sect. 2, the differential equations describing linear non-adiabatic MHD waves are obtained; in Sect. 3, we provide with an approximate analytical solution and with an exact numerical solution for the coupled system of differential equations; in Sect. 4 the results of this study are presented; in Sect. 5 our conclusions are drawn.

\section{Basic equations}

The basic MHD equations for the discussion of linear and nonadiabatic MHD waves are:

$$
\begin{aligned}
& \frac{D \rho}{D t}+\rho \nabla \cdot \boldsymbol{v}=\mathbf{0} \\
& \rho \frac{D \boldsymbol{v}}{D t}=-\nabla p+\frac{1}{\mu}(\nabla \times \boldsymbol{B}) \times \boldsymbol{B} \\
& \frac{D p}{D t}-\frac{\gamma p}{\rho} \frac{D \rho}{D t}+(\gamma-1)[\rho L(\rho, T)-\nabla \cdot(\boldsymbol{\kappa} \cdot \nabla T)]=0 \\
& \frac{\partial \boldsymbol{B}}{\partial t}=\nabla \times(\boldsymbol{v} \times \boldsymbol{B}) \\
& \nabla \cdot \boldsymbol{B}=0 \\
& p=\frac{\rho R T}{\tilde{\mu}}
\end{aligned}
$$

where $\frac{D}{D t}=\frac{\partial}{\partial t}+\boldsymbol{v} \cdot \nabla$ is the material derivative for time variations following the motion. In Eq. (3), the term $\nabla \cdot(\kappa \cdot \nabla T)$ represents the thermal conduction, although in our case thermal conduction perpendicular to the magnetic field $\left(\kappa_{\perp}\right)$ has been neglected and only parallel thermal conduction $\left(\kappa_{\|}\right)$has been taken into account which, for prominence conditions, is given by $\kappa_{\|}=10^{-11} T^{5 / 2} \mathrm{~W} \mathrm{~m}^{-1} \mathrm{~K}^{-1} . L$ is the heat-loss function, which depends on the local plasma parameters, and in the case of an equilibrium with uniform temperature and density, $T_{0}$ and $\rho_{0}$, such as we consider here

$L\left(\rho_{0}, T_{0}\right)=0$.

Usually, in solar applications this function represents the difference between an arbitrary heat input and a radiative loss function which, in our case, has been chosen as the optically 
thin radiative loss function (Hildner 1974). Then, our heat-loss function is given by

$L(\rho, T)=\chi^{*} \rho T^{\alpha}-h \rho^{a} T^{b}$,

$\chi^{*}$ and $\alpha$ being piecewise functions depending on the temperature (Hildner 1974), and imposing condition (7) on Eq. (8) we can obtain the numerical value of the constant $h$ for each considered heating mechanism. The use of an optically thin radiative loss function seems to be a reasonable approach for coronal, or almost coronal, conditions, while it may not be valid for prominence conditions because they are optically thick. In this case, the radiative losses from the internal part of the prominence are greatly reduced and this can be represented by changing the exponent $\alpha$ in the cooling function, for temperatures $T \leq 10^{4} \mathrm{~K}$, from $\alpha=7.4$ to 17.4 (Milne et al. 1979) or $\alpha=30$ (Rosner et al. 1978), as well as by changing $\chi^{*}$ from $1.76 \times 10^{-13}$ to $1.76 \times 10^{-53}$ and to $7.01 \times 10^{-104}$, respectively. Finally, the last term in Eq. (8) represents an arbitrary heating function which can be modified by taking different values for the exponents $a$ and $b$. In our case, different heating scenarios have been considered, and the values taken into account for the exponents $a$ and $b$ in Eq. (8) are (Rosner et al. 1978; Dahlburg \& Mariska 1988)

1. Constant heating per unit volume $(a=b=0)$.

2. Constant heating per unit mass $(a=1, b=0)$.

3. Heating by coronal current disipation $(a=1, b=1)$.

4. Heating by Alfvén mode/mode conversion $(a=b=7 / 6)$.

5. Heating by Alfvén mode/anomalous conduction damping $(a=1 / 2, b=-1 / 2)$.

As our equilibrium configuration we use a homogeneous slab bounded in the transverse direction with a width $2 x_{0}$. Such slab is threaded by a constant transverse magnetic field which we assume anchored in the lateral walls of the slab. The equilibrium magnitudes of the slab are given by

$p_{0}=$ const., $\rho_{0}=$ const., $T_{0}=$ const., $\boldsymbol{B}_{0}=B_{0} \hat{e}_{x}, \boldsymbol{v}_{0}=\mathbf{0}$,

with $B_{0}=$ const. form

Considering small perturbations from the equilibrium in the

$\boldsymbol{B}(t, \boldsymbol{r})=\boldsymbol{B}_{0}+\boldsymbol{B}_{1}(t, \boldsymbol{r}), \quad p(t, \boldsymbol{r})=p_{0}+p_{1}(t, \boldsymbol{r})$,

$\rho(t, \boldsymbol{r})=\rho_{0}+\rho_{1}(t, \boldsymbol{r}), \quad T(t, \boldsymbol{r})=T_{0}+T_{1}(t, \boldsymbol{r}), \quad \boldsymbol{v}(t, \boldsymbol{r})=\boldsymbol{v}_{1}(t, \boldsymbol{r})$

we linearise the basic Eqs. (1)-(6) to obtain

$\frac{\partial \rho_{1}}{\partial t}+\rho_{0} \nabla \cdot \boldsymbol{v}=0$

$\rho_{0} \frac{\partial v}{\partial t}=-\nabla p_{1}+\frac{1}{\mu}\left(\boldsymbol{B}_{0} \cdot \nabla\right) \boldsymbol{B}_{1}-\frac{1}{\mu} \nabla\left(\boldsymbol{B}_{0} \cdot \boldsymbol{B}_{1}\right)$,

$$
\begin{aligned}
\frac{\partial p_{1}}{\partial t}-c_{\mathrm{s}}^{2} \frac{\partial \rho_{1}}{\partial t}= & (\gamma-1)\left(\boldsymbol{B}_{0} \cdot \nabla\right)\left[\frac{\kappa_{\|}}{B_{0}^{2}}\left(\boldsymbol{B}_{0} \cdot \nabla\right) T_{1}\right] \\
& -(\gamma-1)\left(L+\rho_{0} L_{\rho}\right) \rho_{1}-(\gamma-1) \rho_{0} L_{T} T_{1}
\end{aligned}
$$

$\frac{\partial \boldsymbol{B}_{1}}{\partial t}=\nabla \times\left(\boldsymbol{v} \times \boldsymbol{B}_{0}\right)$,

$\nabla \cdot \boldsymbol{B}_{1}=0$

$\frac{p_{1}}{p_{0}}-\frac{\rho_{1}}{\rho_{0}}-\frac{T_{1}}{T_{0}}=0$

where $c_{\mathrm{s}}^{2}=\frac{\gamma p_{0}}{\rho_{0}}$, is the sound speed squared and the factors $L_{\rho}$, $L_{T}$ are

$L_{\rho}=\left(\frac{\partial L}{\partial \rho}\right)_{T}$

and

$L_{T}=\left(\frac{\partial L}{\partial T}\right)_{\rho}$,

with $T$ and $\rho$ held constant, respectively, at the equilibrium state.

Now, taking all $f_{1}(t, \boldsymbol{r})$ to be of the form $f_{1}(x) \mathrm{e}^{i\left(\omega t+k_{y} y+k_{z} z\right)}$, assuming invariance along the $y$-axis $\left(\frac{\partial}{\partial y}=0\right)$, considering only motions and propagation in the $x z$-plane, and eliminating all the perturbed quantities except for $v_{x}, v_{z}$ and $T_{1}$, we end up with the following set of ordinary differential equations

$c_{\mathrm{s}}^{2} \frac{\mathrm{d}^{2} v_{x}}{\mathrm{~d} x^{2}}+\gamma \omega^{2} v_{x}+i k_{z} c_{\mathrm{s}}^{2} \frac{\mathrm{d} v_{z}}{\mathrm{~d} x}-\frac{i \omega c_{\mathrm{s}}^{2}}{T_{0}} \frac{\mathrm{d} T_{1}}{\mathrm{~d} x}=0$,

$v_{\mathrm{A}}^{2} \frac{\mathrm{d}^{2} v_{z}}{\mathrm{~d} x^{2}}+\left[\omega^{2}-k_{z}^{2}\left(v_{\mathrm{A}}^{2}+\frac{c_{\mathrm{s}}^{2}}{\gamma}\right)\right] v_{z}+\frac{i k_{z}}{\gamma} c_{\mathrm{s}}^{2} \frac{\mathrm{d} v_{x}}{\mathrm{~d} x}+\omega c_{\mathrm{s}}^{2} \frac{k_{z}}{\gamma} \frac{T_{1}}{T_{0}}=0$,

$\kappa_{\|} \frac{T_{0}}{p_{0}} \frac{1}{T_{0}} \frac{\mathrm{d}^{2} T_{1}}{\mathrm{~d} x^{2}}-\left(\omega_{T}+\frac{i \omega}{\gamma-1}\right) \frac{T_{1}}{T_{0}}$
$-\left(1+\frac{i \omega_{\rho}}{\omega}\right) \frac{\mathrm{d} v_{x}}{\mathrm{~d} x}-i k_{z}\left(1+\frac{i \omega_{\rho}}{\omega}\right) v_{z}=0$

where $v_{\mathrm{A}}^{2}=\frac{B_{0 x}^{2}}{\mu \rho_{0}}$ is the Alfvén velocity squared and

$\omega_{\rho}=\frac{\rho_{0}}{p_{0}}\left(L+\rho_{0} L_{\rho}\right), \quad \omega_{T}=\frac{\rho_{0}}{p_{0}} L_{T} T_{0}$.

\section{Methods}

Since we are interested in studying the damping time of magnetoacoustic modes in a bounded medium with physical properties akin to those of quiescent prominences, we have chosen the following values for the equilibrium parameters: $B_{0}=10 \mathrm{G}$, $\rho_{0}=5 \times 10^{-11} \mathrm{~kg} \mathrm{~m}^{-3}, T_{0}=8000 \mathrm{~K}, \tilde{\mu}=0.8$ and $x_{0}=3000 \mathrm{~km}$. Furthermore, for our computations the importance of radiation and thermal conduction can be quantified in terms of two dimensionless parameters (De Moortel \& Hood 2004), namely, the thermal ratio,

$d=\frac{(\gamma-1) \kappa_{\|} T_{0} \rho_{0}}{\gamma^{2} p_{0}^{2} \tau_{\mathrm{s}}}=\frac{1}{\gamma} \frac{\tau_{\mathrm{s}}}{\tau_{\text {cond }}}$,

which is $1 / \gamma$ times the ratio of the sound travel time $\left(\tau_{\mathrm{s}}=2 x_{0} / c_{\mathrm{s}}\right)$ to the thermal conduction timescale $\left(\tau_{\text {cond }}=\right.$ $\left.4 x_{0}^{2} p_{0} /\left[(\gamma-1) \kappa_{\|} T_{0}\right]\right)$, and the radiation ratio,

$r=\frac{(\gamma-1) \tau_{\mathrm{s}} \rho_{0}^{2} \chi^{*} T_{0}^{\alpha}}{\gamma p_{0}}=\frac{\tau_{\mathrm{s}}}{\tau_{\mathrm{r}}}$ 
which is the ratio of the sound travel time to the radiation timescale $\left(\tau_{\mathrm{r}}=\gamma p_{0} /\left[(\gamma-1) \rho_{0}^{2} \chi T_{0}^{\alpha}\right]\right)$. Using the above equilibrium parameters, we can compute the sound speed in the prominence as well as parameters $d$ and $r$ and we find that

$c_{\mathrm{s}}=11.7 \mathrm{~km} \mathrm{~s}^{-1}, \quad d=1.04 \times 10^{-6}, \quad r=0.82$.

The above results show that for the chosen parameters the thermal conduction time is much greater than the sound travel time, and when the values of the considered parameters are modified within ranges typical of quiescent prominences the value of $d$ remains of the order of $10^{-7}$. This suggests that thermal conduction does not play a relevant role in our study and can be neglected in our calculations, and that the balance between radiative losses and heating is responsible for the damping of magnetoacoustic modes in the present model.

\subsection{Analytical solution}

The neglect of thermal conduction allows us to obtain analytical solutions for the variables involved in the system of coupled differential Eqs. (16)-(18), as well as an algebraic dispersion relation relating the frequency to the vertical wavenumber. Once ther mal conduction has been neglected, one can substitute $T_{1}$ from Eq. (18) in Eqs. (16) and (17). Then, we obtain two coupled differential equations involving the perturbed velocities. Next, we assume that the solutions for the perturbed velocities can be written as

$v_{x}=A \mathrm{e}^{-i k_{x} x}$,

$v_{z}=C \mathrm{e}^{-i k_{x} x}$,

and following the same procedure as in Oliver et al. (1992) the solutions for the perturbed velocities can be written as

$$
\begin{aligned}
& v_{x}=A_{1} \mathrm{e}^{-i k_{1} x}+A_{2} \mathrm{e}^{-i\left(-k_{1}\right) x}+A_{3} \mathrm{e}^{-i k_{2} x}+A_{4} \mathrm{e}^{-i\left(-k_{2}\right) x}, \\
& v_{z}=C_{1} \mathrm{e}^{-i k_{1} x}+C_{2} \mathrm{e}^{-i\left(-k_{1}\right) x}+C_{3} \mathrm{e}^{-i k_{2} x}+C_{4} \mathrm{e}^{-i\left(-k_{2}\right) x} .
\end{aligned}
$$

Next, we impose the boundary conditions

$v_{x}\left(x_{0}\right)=v_{x}\left(-x_{0}\right)=v_{z}\left(x_{0}\right)=v_{z}\left(-x_{0}\right)=0$

in Eqs. (24) and (25) and look for a nontrivial solution, which provides us with a final and very cumbersome algebraic equation representing the dispersion relation.

Once the coefficients of the dispersion relation have been computed, we are left with a fifth order equation in $\omega$ relating the frequency with the vertical wavenumber, $k_{z}$. This algebraic equation can be solved numerically and, for a fixed real value of $k_{z}$, we look for complex solutions of the frequency $\omega$ expressed as $\omega=\omega_{\mathrm{R}}+\mathrm{i} \omega_{\mathrm{I}}$. From the Appendix in Carbonell et al. (2004), we obtain one purely imaginary root, corresponding to the thermal or condensation mode, and two pair of roots corresponding to the wave modes: one pair representing the slow mode and another pair representing the fast mode. For each pair $\left(\omega_{1}, \omega_{2}\right), \omega_{1}=\omega_{\mathrm{R}}+\mathrm{i} \omega_{\mathrm{I}}$ and $\omega_{2}=-\omega_{\mathrm{R}}+\mathrm{i} \omega_{\mathrm{I}}$. Then, the period of the waves is given by $P=\frac{2 \pi}{\omega_{\mathrm{R}}}$ and the damping time is $\tau_{\mathrm{D}}=\frac{1}{\omega_{\mathrm{I}}}$.

\subsection{Numerical solution}

To solve the full set of coupled differential Eqs. (16)-(18), we have used the code PDE2D (Sewell 2003) based on finite elements. The eigenvalue problem is first written in the linear and homogeneous form. Since the eigenvalue $\omega$ is in general a complex number the equations are separated into their real and imaginary parts. Cubic Hermite elements are used, which provide an appropriate description of slow and fast modes. The program uses a collocation method and the generalized matrix eigenvalue problem is solved using the shifted inverse power method, which finds the eigenvalue closest to the initial frequency guess and the corresponding eigenfunction. Since in this case thermal conduction is taken into account, a comparison with the already found analytical solution will point out if our neglect of thermal conduction is correct or not.

\section{Results}

We have first compared the results obtained from the analytical and numerical solutions. The agreement between both solutions is so excellent that the differences between analytical and numerical periods and damping times are of the order of $10^{-6}$. This indicates that the analytical solution of the coupled system of differential equations obtained by neglecting thermal conduction represents with great accuracy the behaviour of the perturbed quantities, as well as the period and damping time of the magnetoacoustic modes of oscillation, and confirms the lack of relevance of thermal conduction in our problem. Taking into account this agreement between the analytical and numerical solutions, the figures shown below correspond to the results obtained with the numerical solution which, furthermore, shows that the perturbed velocities display the same behaviour as in Oliver et al. (1992), i.e. the horizontal component of the perturbed velocity is dominant for the slow modes while the opposite happens for the fast modes.

Figures 1a-d displays the variation of the period and damping time of the fundamental slow and fast mode as functions of the vertical wavelength, when different heating mechanisms are considered. It can be seen that the periods and damping times of slow modes are independent of the vertical wavelength and that, for the interval of observationally determined wavelengths, the damping time of fast modes is much greater than that of slow modes. For this reason, in the following we will only consider slow modes. Moreover, for a fixed vertical wavelength, and when different heating mechanims are considered, the period of slow modes is almost unaffected while for the damping time the range of variation is more important, and such as can be expected, attaining the smallest value when no heating is considered. On the other hand, for the chosen values of the parameters describing prominence physical properties, and the different heating mechanisms considered, the periods are smaller than the damping times.

Figures $2 \mathrm{a}-\mathrm{d}$ show, for a fixed vertical wavelength and different heating mechanisms, the variation of the period and the damping time of the fundamental slow mode versus density and temperature. Figures $2 \mathrm{a}$ and $2 \mathrm{c}$ show that for all the considered heating mechanisms, even in the case of no heating $(h=0)$, the 

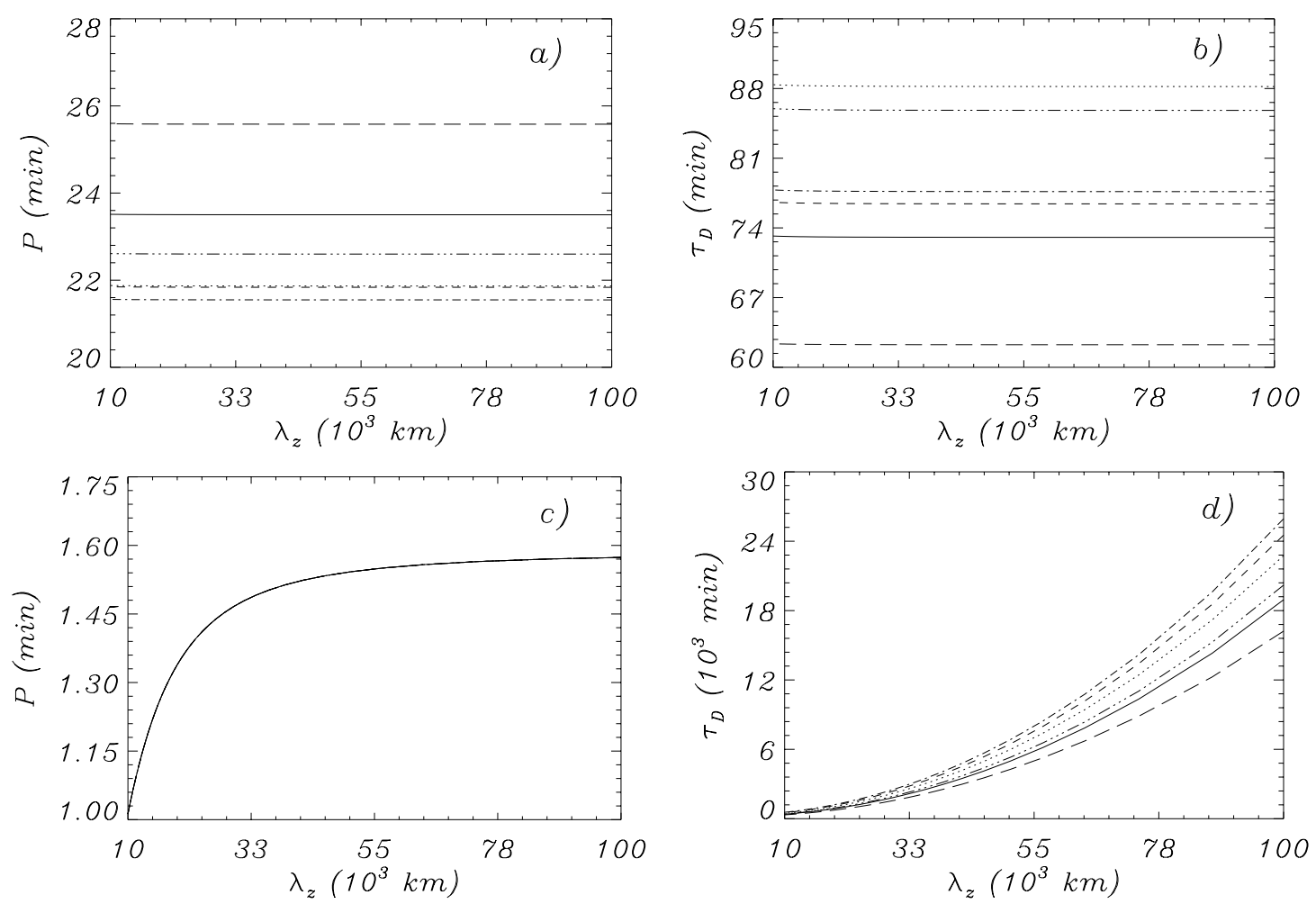

Fig. 1. Variation with the vertical wavelength $\left(\lambda_{z}\right)$ of the period $(P)$ and damping time $\left(\tau_{\mathrm{D}}\right)$ for the fundamental slow (upper panels) and fast (lower panels) magnetoacoustic modes, for different heating mechanisms: dotted line $(a=1, b=0)$; three dot-dashed $(a=0.5, b=-0.5)$; dashed-dotted ( $a=b=7 / 6)$; short-dashed line $(a=b=1)$; continuous line $(a=b=0)$; long-dashed line $(h=0)$. In panel c) only one curve is shown since the period of fast modes is unaffected by the different heating mechanisms.

numerical value of the period of the slow modes suffers slight variations with temperature or density. However, the change in the numerical value of the damping time (Figs. $2 b$ and $2 d$ ) is more significant. In Figs. $1 \mathrm{a}$ and $1 \mathrm{~b}$ we have seen that for the standard values of prominence parameters, the fundamental slow mode period is smaller than the damping time (23 and $73 \mathrm{~min}$, respectively), but when the prominence temperature is of the order of $6000 \mathrm{~K}$, Fig. $2 \mathrm{~d}$ shows that the period is greater than the damping time (23 and $19 \mathrm{~min}$, respectively), and the same happens when the density is decreased. Then, depending on the physical properties of the prominence, the period of oscillation of the fundamental slow mode can be greater or smaller than the damping time, so, small or strong attenuation of the oscillation can be obtained.

Now, in order to try to understand the above variations of the damping times with temperature and density, we study the behaviour of the damping time with respect to the radiation timescale. Figures $3 \mathrm{a}$ and $3 \mathrm{~b}$ show, for a particular heating mechanism and wavelength, the behaviour of the damping time of the fundamental slow mode versus the radiation timescale when the temperature or density are varied. In both cases the behaviour is similar, the damping time first decreases, goes through a minimum at certain temperature or density $\left(T_{0} \sim 5909 \mathrm{~K}, \rho_{0} \sim 7.9 \times 10^{-12} \mathrm{~kg} \mathrm{~m}^{-3}\right)$ and starts to grow again with $\tau_{\mathrm{r}}$. In the left branch of the curve, away from the minimum, we are close to the isothermal limit ( $\tau_{\mathrm{r}}$ tends to zero), while in the right branch, also away fron the minimum, we are close to the adiabatic limit ( $\tau_{\mathrm{r}}$ tends to infinity). Around the minimum there is a zone of transition between both regimes and it is seen that the same damping time, corresponding to the adiabatic or isothermal branch of the curve, can be obtained for different temperatures or densities. This behaviour of the damping time versus the radiation timescale is similar to that found by Terradas et al. (2001) in their study of the radiative damping of the slow magnetoacoustic modes in the KippenhahnSchlüter and Menzel's prominence models using Newton's law of cooling and the radiative relaxation time. Taking into account the interval of values we are considering for the temperature and density, Figs. $2 b$ and $2 d$ correspond to the branch of the curve $\tau_{\mathrm{D}}$ versus $\tau_{\mathrm{r}}$ to the left of the minimum, i.e. we are in the isothermal zone, away from the adiabatic limit.

The case of low radiative losses in the prominence (optically thick) can be represented by modifying the values of parameters $\chi^{*}$ and $\alpha$. Figures $4 \mathrm{a}$ and $4 \mathrm{~b}$ show, for a particular heating mechanism and wavelength, the behaviour of the damping time of the fundamental slow mode versus the radiation timescale, when the temperature or density are varied, for radiative losses characterized by $\chi^{*}=1.76 \times 10^{-53}, \alpha=$ 17.4. The damping time displays the same behaviour as before and the only difference is that when the temperature is varied the minimum appears at a temperature of $7750 \mathrm{~K}$, while when the density is varied the minimum appears at a density of $3.76 \times 10^{-11} \mathrm{~kg} \mathrm{~m}^{-3}$. Both values are well inside the range of temperatures and densities typical of prominences. Also, combining the values of both parameters a damping time close to the period can be obtained. When radiative losses are reduced 

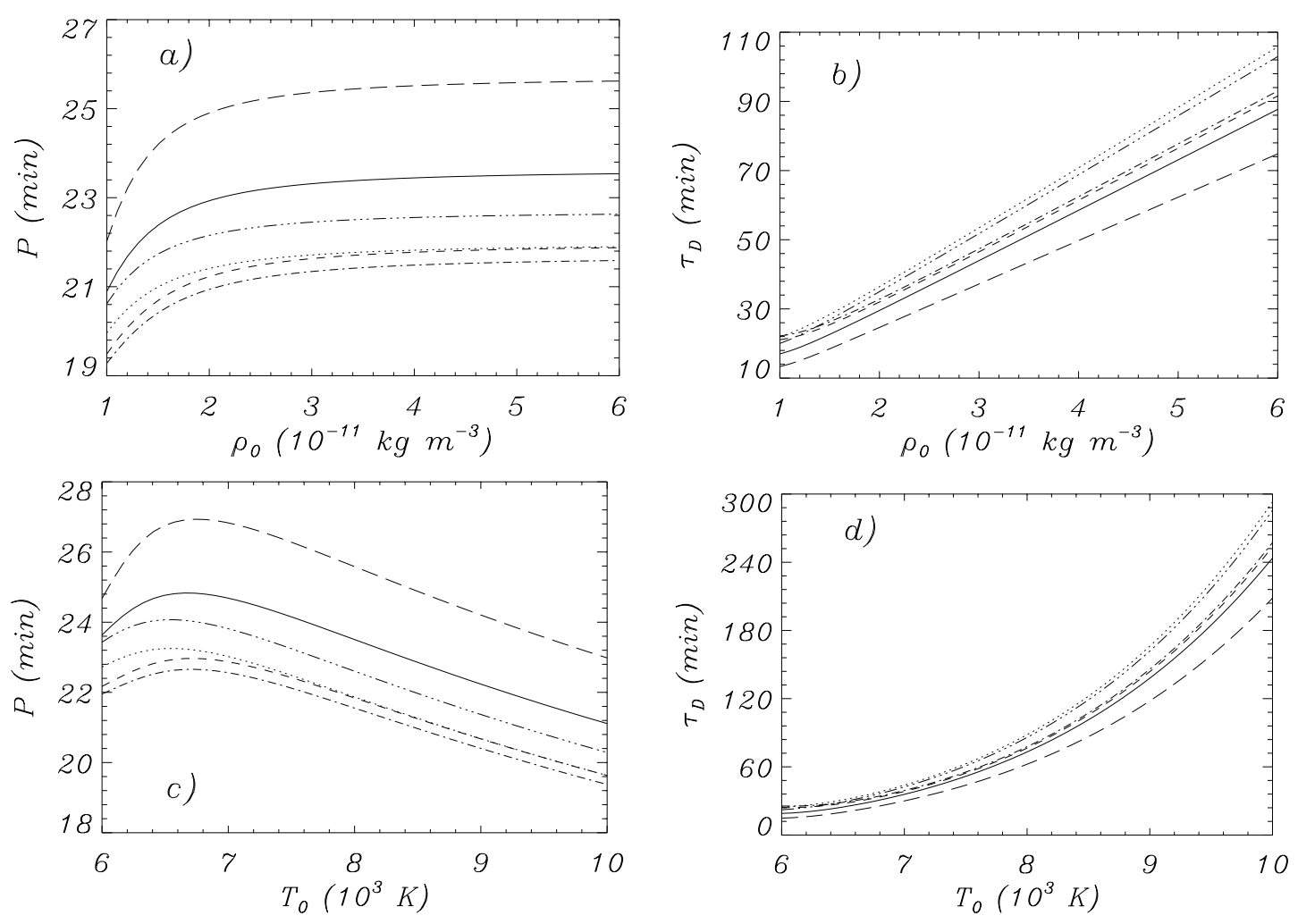

Fig. 2. Fundamental slow mode: variation of the period $(P)$ and damping time $\left(\tau_{\mathrm{D}}\right)$ versus temperature and density, for different heating mechanisms: dotted line $(a=1, b=0)$; three dot-dashed $(a=0.5, b=-0.5)$; dashed-dotted $(a=b=7 / 6)$; short-dashed line $(a=b=1)$; continuous line $(a=b=0)$; long-dashed line $(h=0)]$. In all cases $\lambda_{z}=18840 \mathrm{~km}$.
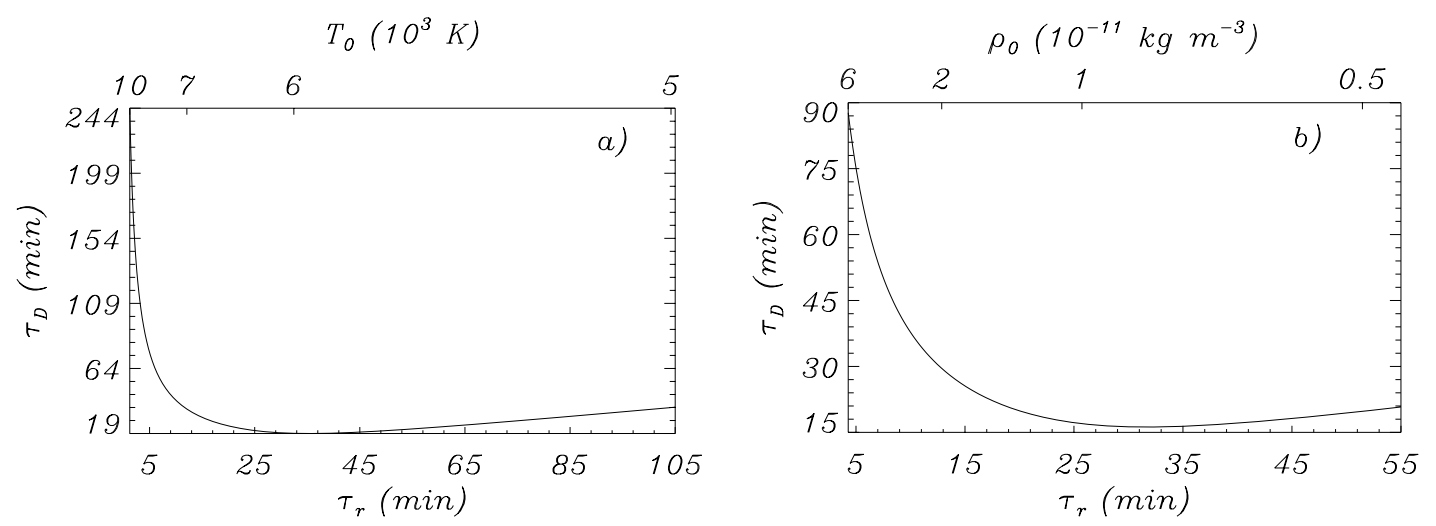

Fig. 3. Fundamental slow mode: variation of the damping time $\left(\tau_{\mathrm{D}}\right)$ versus the radiation timescale $\left(\tau_{\mathrm{r}}\right)$ for a constant heating per unit volume ( $a=b=0$ ) and $\lambda_{z}=18840 \mathrm{~km}$. a) The upper axis shows the interval of temperatures considered; b) the upper axis shows the interval of densities considered.

still more by taking $\chi^{*}=7.01 \times 10^{-104}, \alpha=30$, Fig. 5a shows a similar behaviour as Fig. $4 \mathrm{a}$, with the minimum appearing again at a typical prominence temperature $(8500 \mathrm{~K})$, while in the case of the density (Fig. 5b) the minimum appears at a much larger value $\left(\rho_{0}=3 \times 10^{-10} \mathrm{~kg} \mathrm{~m}^{-3}\right)$ than that of a quiescent prominence. In both cases, the damping time never attains a value smaller than that of the period.

On the other hand, when radiative losses are reduced and the density is kept constant, the minimum of the damping time appears at a low $\tau_{\mathrm{r}}$ while when the temperature is kept constant it appears at a larger $\tau_{\mathrm{r}}$ (Figs. 4a,b and Figs. 5a,b). Figures $6 a-c$ show the variation of the damping time with the temperature and density for the three prominence regimes considered and, as a general rule, the part of the surface located to the left of the minimum is the isothermal zone while that to the right is the adiabatic zone. Also, these figures show that within the range of values considered for the temperature and density the size of the isothermal zone is decreased when radiative losses are reduced. The transition from the adiabatic to the isothermal regime can be characterized following the behaviour of temperature perturbations. In the hydrodynamical case, Mihalas \& Mihalas (1984) pointed out that according to Newtonian cooling theory, in the limit of short radiative relaxation times radiative exchange obliterates temperature 

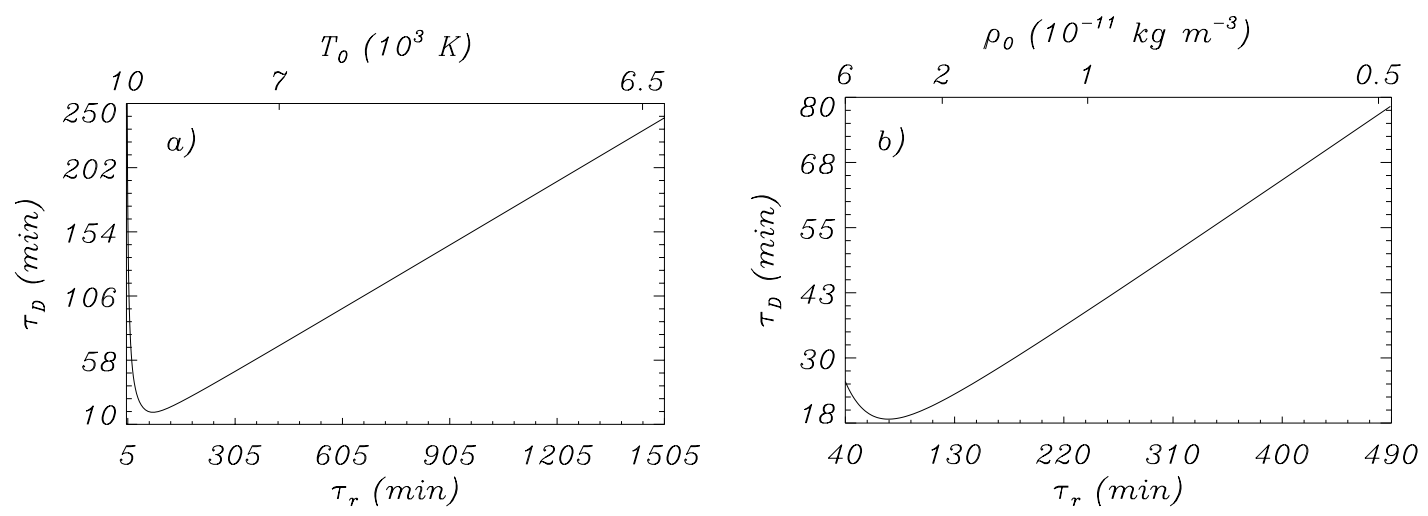

Fig. 4. Fundamental slow mode: variation of the damping time $\left(\tau_{\mathrm{D}}\right)$ versus the radiation timescale $\left(\tau_{\mathrm{r}}\right)$ for a constant heating per unit volume $(a=b=0)$ and $\lambda_{z}=18840 \mathrm{~km}$. a) The upper axis shows the interval of temperatures considered; b) the upper axis shows the interval of densities considered.
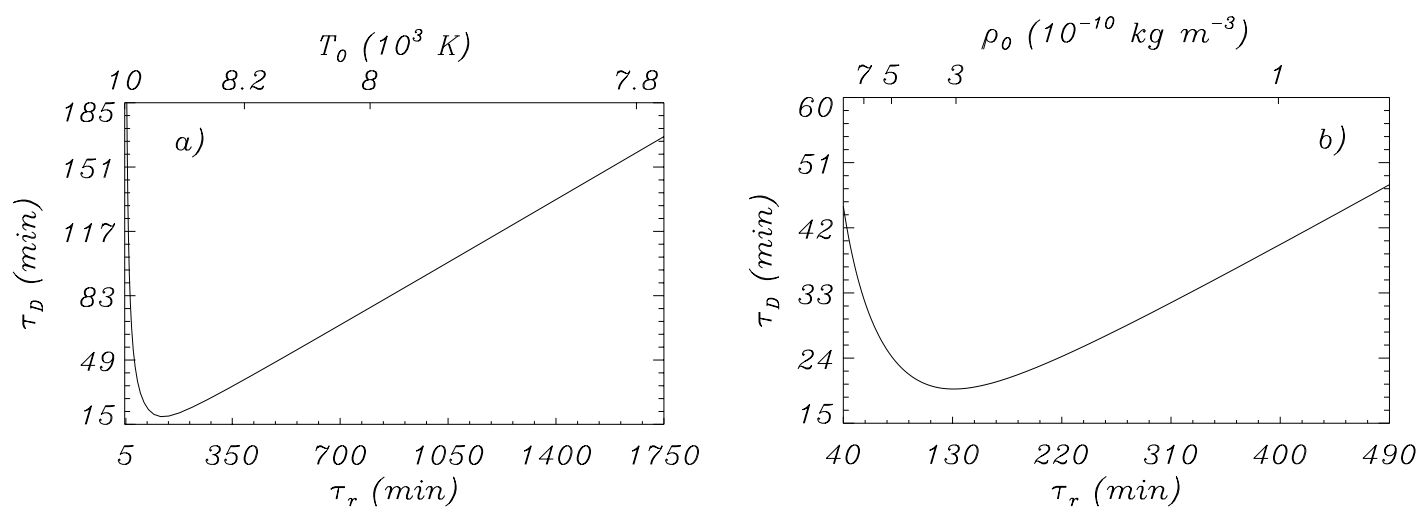

Fig. 5. Fundamental slow mode: Damping time $\left(\tau_{\mathrm{D}}\right)$ versus the radiation timescale $\left(\tau_{\mathrm{r}}\right)$ for a constant heating per unit volume $(a=b=0)$ and $\lambda_{z}=18840 \mathrm{~km}$. a) The upper axis shows the interval of temperatures considered; b) the upper axis shows the interval of densities considered.

fluctuations and accoustic waves propagate isothermally. In our case, the radiation timescale decreases when temperature or density are increased and the transition from the adiabatic to isothermal regime occurs for relatively small values of the radiation timescale (see Figs. 3-5). As a further confirmation, Figs. $7 \mathrm{a}-\mathrm{c}$ show the behaviour of the normalized maximum value of the temperature perturbation versus temperature and density for the different prominence regimes considered, showing a severe decrease of temperature fluctuations coincident with the apparition of the isothermal region shown in Figs. 6a-c.

Finally, Table 1 shows a comparison of the periods and damping times of the slow fundamental mode for the different prominence regimes characterized by the different values of $\chi^{*}$ and $\alpha$. Going from top to bottom the radiative losses are reduced, but the period is only slightly modified. However, comparing the damping times of the different regimes we can see that it diminishes when going from the first to the second regime, and grows again in the case of the third regime. This behaviour of the damping time for the different regimes can again be understood by looking at Figs. 6a-c.

\section{Conclusions}

In this paper, we have analyzed the behaviour of non-adiabatic MHD waves in a homogeneous, isothermal and bounded slab, with physical properties akin to those of quiescent solar prominences. First of all, we have considered the different timescales involved in the problem and have found that, for typical prominence conditions, the thermal conduction timescale is much greater than the sound travel time, so thermal conduction plays no relevant role in the damping of the magnetoacoustic waves and can be neglected. This suggests that the balance between radiative losses and heating is responsible for the damping of oscillations. Analytical and numerical solutions, showing an excellent agreement, have been found, and the main conclusions which can be extracted are:

1. Only slow magnetoacoustic modes show significant time damping, due to the inclusion of non-adiabatic effects, and it is independent of the vertical wavelength.

2. The damping time of the fundamental slow magnetoacoustic mode varies slightly with the heating mechanism considered, attaining its lowest value for the case of no heating.

3. When the prominence density (temperature) is kept constant, the damping time of the fundamental slow magnetoacoustic mode increases with the temperature (density) when an interval of temperature (density) values typical of quiescent prominences is considered.

4. In the case of the fundamental slow mode, and for the considered values of prominence temperature, density and magnetic field (see Table 1), the period is around $25 \mathrm{~min}$ 


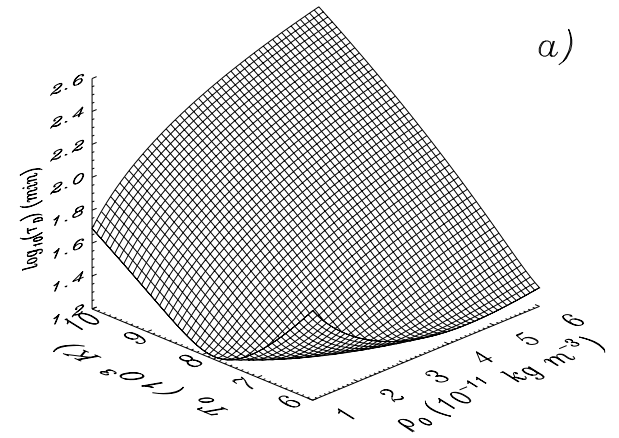

b)

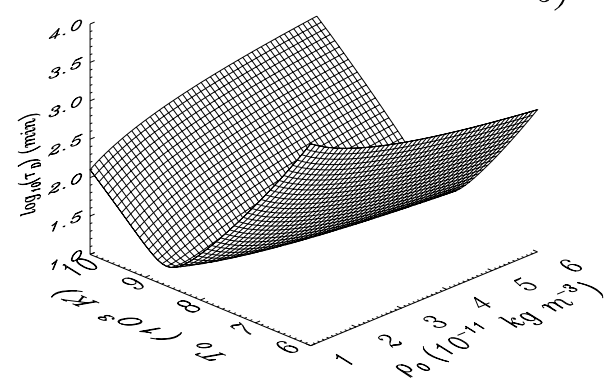

c)

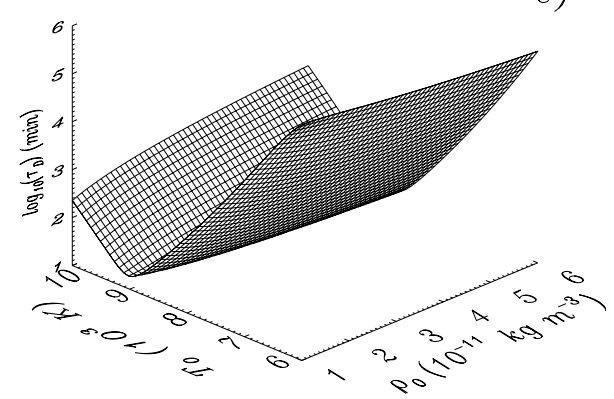

Fig. 6. Fundamental slow mode: damping time $\left(\tau_{\mathrm{D}}\right)$ versus the temperature and density, for a constant heating per unit volume $(a=$ $b=0)$ and $\lambda_{z}=18840 \mathrm{~km}$. a) Prominence regime characterized by $\chi^{*}=1.76 \times 10^{-13}, \alpha=7.4$; b) prominence regime characterized by $\chi^{*}=1.76 \times 10^{-53}, \alpha=17.4$; c) prominence regime characterized by $\chi^{*}=7.01 \times 10^{-104}, \alpha=30$.

Table 1. Period and damping times of the slow fundamental mode for different values of $\chi^{*}$ and $\alpha$ (MKS units). $B_{0}=10 \mathrm{G}, \rho_{0}=5 \times$ $10^{-11} \mathrm{~kg} \mathrm{~m}^{-3}, T_{0}=8000 \mathrm{~K}, \lambda_{z}=18840 \mathrm{~km}$.

\begin{tabular}{cccc}
\hline \hline$\chi^{*}$ & $\alpha$ & $P(\min )$ & $\tau_{\mathrm{D}}(\min )$ \\
\hline $1.76 \times 10^{-13}$ & 7.4 & 23 & 73 \\
$1.76 \times 10^{-53}$ & 17.4 & 21 & 23 \\
$7.01 \times 10^{-104}$ & 30 & 17 & 77 \\
\hline
\end{tabular}

while the damping time is around $73 \mathrm{~min}$. However, when no heating is considered and the prominence temperature is reduced to $T_{0}=6000 \mathrm{~K}$, the period of the slow fundamental mode is of the same order while the damping time decreases to $19 \mathrm{~min}$.

5. When radiative losses in the prominence are reduced, trying to mimic the optically thick case (or less optically thin case), and temperatures and densities typical of prominences are considered, the damping time of slow modes a)

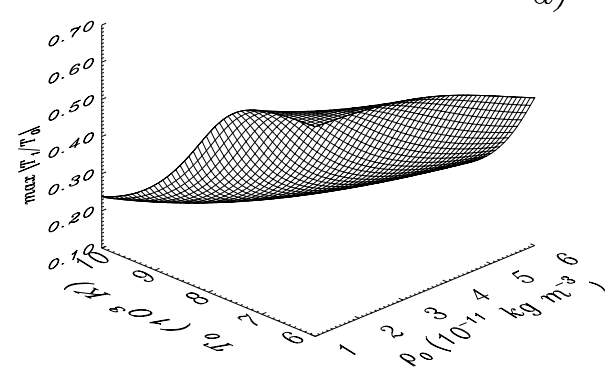

b)

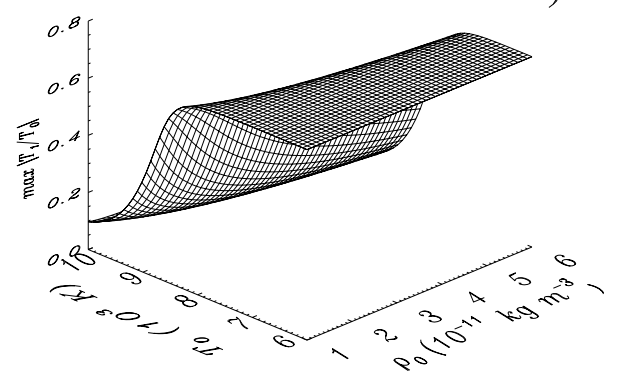

c)

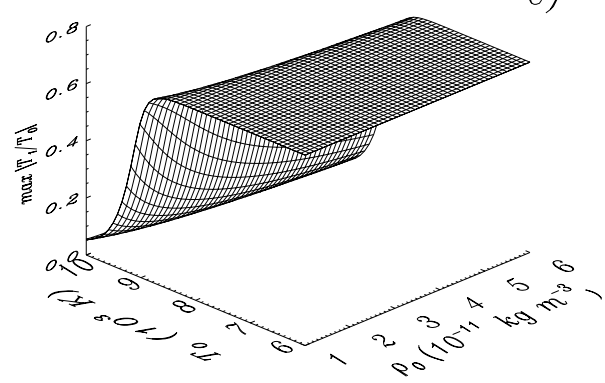

Fig. 7. Variation of the normalized temperature perturbation versus the temperature and density, for a constant heating per unit volume $(a=b=0)$ and $\lambda_{z}=18840 \mathrm{~km}$. a) Prominence regime characterized by $\chi^{*}=1.76 \times 10^{-13}, \alpha=7.4$; b) prominence regime characterized by $\chi^{*}=1.76 \times 10^{-53}, \alpha=17.4$; c) prominence regime characterized by $\chi^{*}=7.01 \times 10^{-104}, \alpha=30$.

changes its behaviour with respect to the optically thin case. This behaviour can be understood by looking at the variation of the damping time versus the radiation timescale, which shows that when the temperature or density are varied we move from the adiabatic to the isothermal limit. In general, in all the variations of the damping time with the density or temperature, the branch of the curve to the left of the minimum comes from the isothermal limit while the right branch goes to the adiabatic limit. In the optically thick cases, it seems impossible to obtain damping times smaller than the period, for temperatures or densities typical of prominences. However, when considering this situation we must keep in mind that we are considering the whole body of the prominence as optically thick, when in fact only the most internal part of the prominence seems to behave in this way. Probably, an intermediate configuration would better represent the actual situation in prominences. In all the cases considered, the transition from the adiabatic to the isothermal regime is characterized by a significant decrease of the value of the temperature perturbation. 
6. When the temperature and density of the prominence are modified simultaneously, the damping time varies in a complex way which also depends on the prominence regime considered. In all the prominence regimes, a minimum of the damping time can be obtained for a certain value of temperature and density.

7. A clear distinction between the adiabatic and isothermal regimes can be made by taking into account the behaviour of temperature fluctuations with the radiation timescale. For short radiation timescales, temperature fluctuations become very small characterizing the isothermal regime.

Terradas et al. (2001) studied the damping of the oscillations of a $K-S$ prominence model using Newton's law of cooling. They found that the fundamental slow mode, with a period similar to that of our case, was almost undamped. However, in our case, using a more complete energy equation, we show that it is possible to obtain short and long damping times for the fundamental slow mode, that thermal conduction does not play any role and that the heating mechanism does not have a strong influence on the damping time or period.

On the other hand, the simplicity of the model used to represent the quiescent prominence, a structureless slab, prevents long periods and damping times from being obtained simultaneously. New and interesting physical results can be obtained by the addition of a surrounding medium with the physical properties of the solar corona. In this case, the interplay of the prominence, where radiation is the relevant damping mechanism, with the solar corona, in which thermal conduction will be responsible for damping, can generate interesting results and we will be able to obtain long periods, and probably longer damping times as well. On the other hand, the consideration of two-dimensional models such as Menzel's model or the more complete Hood-Anzer (Hood \& Anzer 1990), which includes a coronal arcade, should also provide us with a more complete description of the damping of oscillations in quiescent prominences via the thermal damping of magnetoacoustic modes.

Acknowledgements. The authors acknowledge the financial support received from MCyT under grant AYA2003-00123.

\section{References}

Ballai, I. 2003, A\&A, 410, L17

Ballester, J. L. 2003, in NATO Advanced Research Workshop on Turbulence, Waves and Instabilities in the Solar Plasma, ed. R. Erdélyi, K. Petrovay, B. Roberts, \& M. Aschwanden (The Netherlands: Kluwer Academic Publishers), 193

Bashkirtsev, V. S., \& Mashnich, G. P. 1984, Sol. Phys., 91, 93

Carbonell, M., Oliver, R., \& Ballester, J. L. 2004, A\&A, 415, 739

Dahlburg, R. B., \& Mariska, J. T. 1988, Sol. Phys., 117, 51

De Moortel, I., \& Hood, A. W. 2004, A\&A, 415, 705
Engvold, O. 2001, in INTAS Workshop on MHD Waves in Astrophysical Plasmas, ed. J. L. Ballester, \& B. Roberts (Spain: Universitat de les Illes Balears), 123

Engvold, O. 2004, in Multiwavelength Investigations of Solar Activity (Cambridge University P.), IAU Symp., 233, in press

Field, G. B. 1964, ApJ, 142, 531

Hildner, E. 1974, Sol. Phys., 35, 123

Hood, A. W., \& Anzer, U. 1990, Sol. Phys., 126, 117

Ibañez, M. H., \& Escalona, O. B. 1993, ApJ, 415, 335

Joarder, P. S., \& Roberts, B. 1992, A\&A, 261, 625

Kippenhahn, R., \& Schlüter, A. 1957, ZAp, 43, 36

Laing, G., \& Edwin, P. 1994, Sol. Phys., 151, 191

Landman, D. A., Edberg, S. J., \& Laney, C. D. 1977, ApJ, 218, 888

Menzel, D. H., \& Evans, J. W. 1953, Acad. Naz. Lincei. Conv. Volta, 11,119

Mihalas, D., \& Mihalas, B. W. 1984, in Foundations of Radiation Hydrodynamics (New York: Oxford University Press)

Priest, E. R., Milne, A. M., \& Roberts, B. 1979, ApJ, 232, 304

Molowny-Horas, R., Oliver, R., Ballester, J. L., \& Baudin, F. 1997, Sol. Phys., 172, 181

Molowny-Horas, R., Wiehr, E., Balthasar, H., Oliver, R., \& Ballester, J. L. 1999, JOSO Annual Report 1998. Astronomical Institute Tatranska Lomnica, 126

Oliver, R. 1999, in Magnetic fields and Solar Processes, ESA SP-448, 425

Oliver, R. 2001, in INTAS Workshop on MHD Waves in Astrophysical Plasmas, Universitat de les Ills Balears, 133

Oliver, R. 2004, in SOHO 13 - Waves, Oscillations and Small Scale Transient Events in the Solar Atmosphere: A Joint View from SOHO and TRACE, ESA SP-547, 175

Oliver, R., Ballester, J. L., Hood, A. W., \& Priest, E. R. 1992, ApJ, 400, 369

Oliver, R., Ballester, J. L., Hood, A. W., \& Priest, E. R. 1993, ApJ, 409, 809

Oliver, R., \& Ballester, J. L. 2002, Sol. Phys., 206, 45

Porter, L. J., Klimchuk, J. A., \& Sturrock, P. A. 1994a, ApJ, 435, 482

Porter, L. J., Klimchuk, J. A., \& Sturrock, P. A. 1994b, ApJ, 435, 502

Ramsey, H. E., \& Smith, S. F. 1966, AJ, 71, 197

Regnier, S., Solomon, J., \& Vial, J. C. 2001, ApJ, 376, 292

Rosner, R., Tucker, W. H., \& Vaiana, G. S. 1978, ApJ, 220, 643

Sewell, G. 2003, Finite Differences, Finite Elements and PDE2D

Sutterlin, P., Wiehr, E., Bianda, M., \& Kuveler, G. 1997, A\&A, 321, 921

Terradas, J., Oliver, R., \& Ballester, J. L. 2001, A\&A, 378, 635

Terradas, J., Molowny-Horas, R., Wiehr, E., et al. 2002, A\&A, 393, 637

Thompson, W. T., \& Schmieder, B. 1991, A\&A, 243, 501

Tsubaki, T., \& Takeuchi, A. 1986, Sol. Phys., 104, 313

Webb, A. R., \& Roberts, B. 1980, Sol. Phys., 68, 71

Wiehr, E., Balthasar, H., \& Stellmacher, G. 1989, Hvar Obs. Bull., 13, 131

Wiehr, E. 2004, in SOHO 13 - Waves, Oscillations and Small Scale Transient Events in the Solar Atmosphere: A Joint View from SOHO and TRACE, ESA SP-547, 185

Yi, Z., Engvold, O., \& Keil, S. L. 1991, Sol. Phys., 132, 63 